\title{
Three dimensional laser-assisted atom-probe tomography: Advanced analysis of Si nanostructures
}

Keith Thompson, David J. Larson, Joseph H. Bunton, Robert M. Ulfig, Ty J. Prosa, Jason T. Sebastian, Daniel R. Lenz, Stephan S.A. Gerstl, David A. Reinhard, Jesse D. Olson, Thomas F. Kelly Imago Scientific Instruments, 6300 Enterprise Ln. Suite 100 Madison, WI 53719

Recent advances in three-dimensional atom-probe tomography have transformed the traditional atom-probe into a system capable of analyzing Si-based semiconductor structures of interest that are extracted directly from the $\mathrm{Si}$ wafer in an expedient manner. These advances include: (1) the introduction of a localized counter-electrode geometry [1]; (2) the addition of a laser-assisted ionization and evaporation scheme [2]; and (3) the implementation of a focused ion beam (FIB) liftout and extract sample preparation methodology $[3,4]$.

The introduction of the local electrode has localized the fields present during the analysis. The result is that the analysis time is reduced from days to hours and the analysis field of view is increased to $100 \mathrm{~nm}$ in diameter. In addition, the localizing of field lines implies that closely spaced specimens can be analyzed in turn. The clear implication is that a large number of specimens can now be mounted to a specialized local electrode atom-probe (LEAP) microtip array, Fig. 1. The LEAP microtip array accelerates both sample preparation and atom-probe analysis.

The addition of the laser pulsing mechanism has removed the electrical conductivity requirements that had previously prohibited the analysis of high resistivity materials such as lightly doped Si or thin dielectrics. This has enabled applications such as: the mapping of dopant atom distributions in three dimensions, Fig. 2, accurate composition profiling of silicide structures and SiGe multilayers, Fig. 3, buried interface analysis, Fig. 4, and thin dielectric analysis, Fig. 5.

Finally, the development of a FIB lift-out sample preparation methodology has enabled the straightforward preparation of specimens extracted directly from a specific site on a Si wafer [3,4]. This methodology parallels similar developments in TEM sample preparation. In addition, this process includes a final clean-up step that utilizes a low energy (i.e. 2 or $5 \mathrm{kV}$ ) Ga ${ }^{+}$beam to remove most of the amorphized region that is created during the mill sharpening of the specimen tip [5]. This cleanup step reduces the amorphization depth from $\sim 25 \mathrm{~nm}$ with the standard $30 \mathrm{kV}$ beam energy to $<2$ $\mathrm{nm}$ with the $2 \mathrm{kV}$ low energy beam.

[1] T.F. Kelly, et al Microscopy and Microanalysis, 10 (2004) 373.

[2] J.H. Bunton, et. al. "Instrumentation Developments in Atom Probe Tomography: Applications in Semiconductor Research," this proceeding.

[3] M. K. Miller et al., Ultramicroscopy 102 (2005) 287.

[4] D. Lawrence, K. Thompson, D.J. Larson, "Site-specific lift out sample preparation technique for atom probe analysis," this Proceeding.

[5] K. Thompson, B. Gorman, D. J. Larson, Brandon van Leer, Liang Hong, "Minimization of Ga Induced FIB Damage Using Low Energy Clean-up," this Proceeding. 

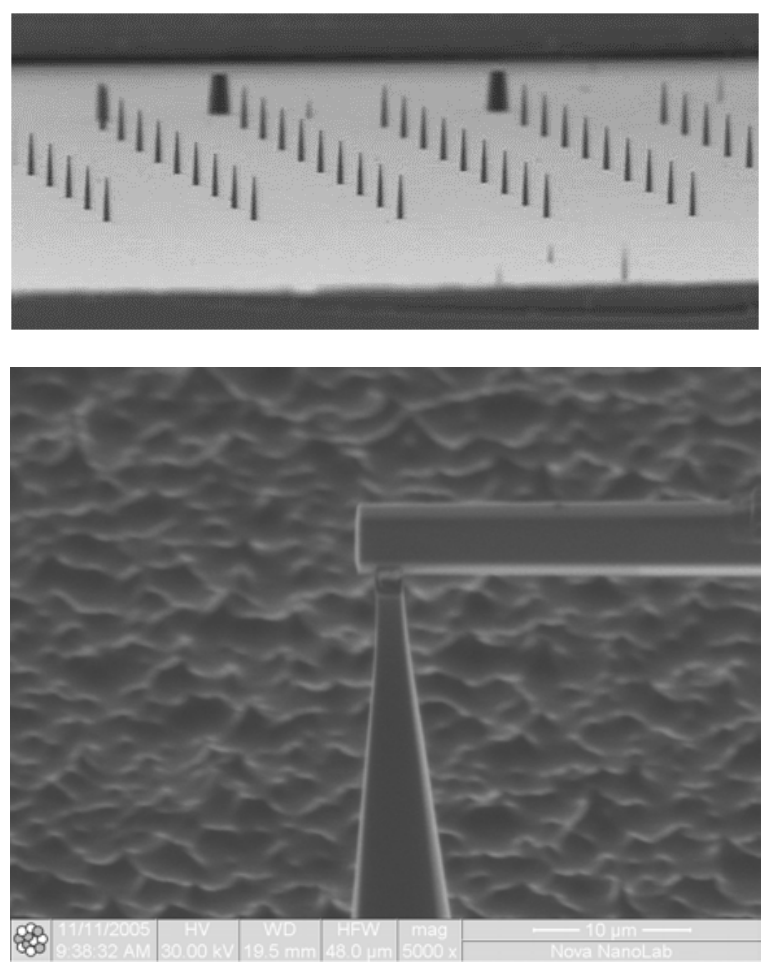

Fig. 1a. (top) LEAP microtip array

Fig. 1b. (btm) Sample wedge aligned to microtip post.

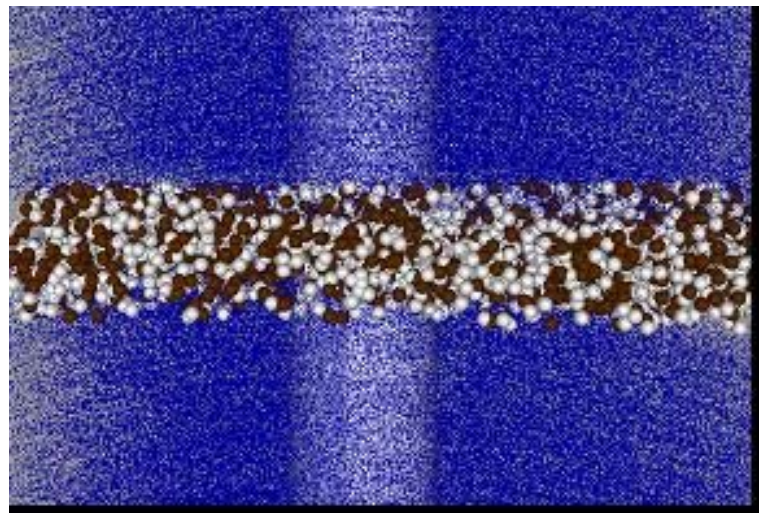

Figure 6. 3-D atom map of a $\mathrm{Si} / \mathrm{HfSiO} / \mathrm{Si}$ structure.
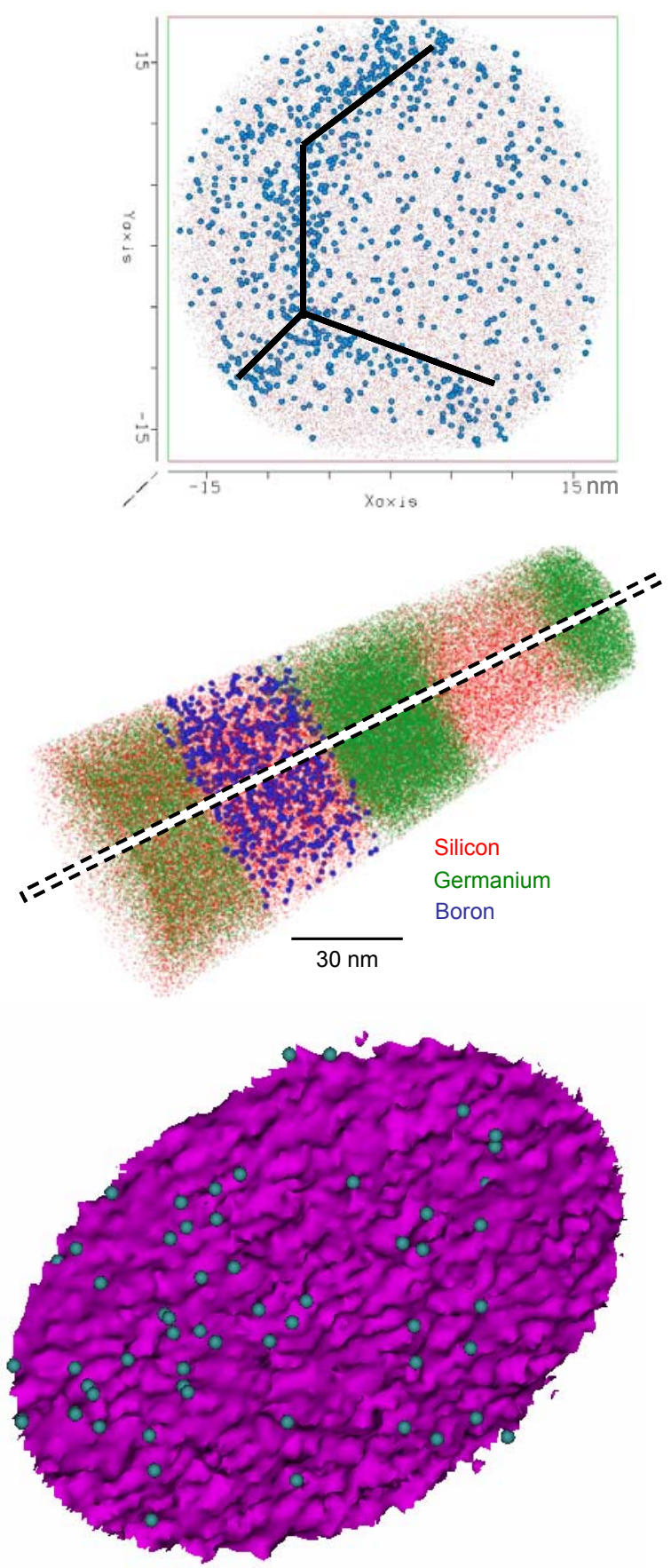

Figure 2. B distribution in poly-Si

Figure 3. SiGe multilayer

Figure 4. SiGe interface decorated with B. 